\title{
PromBase: a web resource for various genomic features and predicted promoters in prokaryotic genomes
}

\author{
Vetriselvi Rangannan and Manju Bansal
}

\begin{abstract}
Background: As more and more genomes are being sequenced, an overview of their genomic features and annotation of their functional elements, which control the expression of each gene or transcription unit of the genome, is a fundamental challenge in genomics and bioinformatics.

Findings: Relative stability of DNA sequence has been used to predict promoter regions in 913 microbial genomic sequences with GC-content ranging from $16.6 \%$ to $74.9 \%$. Irrespective of the genome GC-content the relative stability based promoter prediction method has already been proven to be robust in terms of recall and precision. The predicted promoter regions for the 913 microbial genomes have been accumulated in a database called PromBase. Promoter search can be carried out in PromBase either by specifying the gene name or the genomic position. Each predicted promoter region has been assigned to a reliability class (low, medium, high, very high and highest) based on the difference between its average free energy and the downstream region. The recall and precision values for each class are shown graphically in PromBase. In addition, PromBase provides detailed information about base composition, CDS and CG/TA skews for each genome and various DNA sequence dependent structural properties (average free energy, curvature and bendability) in the vicinity of all annotated translation start sites (TLS).

Conclusion: PromBase is a database, which contains predicted promoter regions and detailed analysis of various genomic features for 913 microbial genomes. PromBase can serve as a valuable resource for comparative genomics study and help the experimentalist to rapidly access detailed information on various genomic features and putative promoter regions in any given genome. This database is freely accessible for academic and non- academic users via the worldwide web http://nucleix.mbu.iisc.ernet.in/prombase/.
\end{abstract}

\section{Introduction}

Controlling gene expression is the central process in all cellular processes. The synchronized control of gene expression is accomplished by the interplay of multiple regulatory mechanisms. Promoter elements are the key regulatory regions, which recruit the transcriptional machinery through the binding of a variety of regulatory proteins to the short oligonucleotide sequences occurring within them. Since these transcriptional regulatory elements are often short and degenerate, their identification in bacterial genomes is a difficult problem. As a consequence of large-scale genome sequencing methods and

\footnotetext{
* Correspondence: mb@mbu.iisc.ernet.in
Molecular Biophysics Unit, Indian Institute of Science, Bangalore-560 012,

* Correspondence: mb@mbu.iisc.ernet.in
Molecular Biophysics Unit, Indian Institute of Science, Bangalore-560 012, India
}

high throughput technologies, vast amount of DNA sequence data has accumulated within last decade [1]. Hence, it is essential to have highly reliable rapid annotation of functional elements, especially those responsible for controlling gene expression in organisms, since there has been only limited experimental investigation. The traditional genetic, biochemical techniques available to identify and characterize promoter regions are not readily scalable to probe whole genomes and cannot meet the challenge of the genomic era.

There are few model organisms which have been systematically annotated for promoter regions and for regulatory binding sites and curated into public domain databases. RegulonDB, Ecocyc and PromEC are the genome specific resources for E. coli, while DBTBS and 
MtbRegList provide information about B. subtilis and $M$. tuberculosis genomes respectively [2-6]. Recently, whole genome expression profiles have led to characterization of bacterial and archaeal transcriptomes [7-10]. Apart from the genome specific databases mentioned above, several databases involve human expertise to handle the annotations and summarize subsets of data related to different aspects of bacterial regulation. PRODORIC database provides information about operon, promoter structures, transcription factor binding sites and their position weight matrix (PWM) in prokaryotes with focus on pathogenic organisms which were collected and screened manually from the original scientific literature [11]. Tractor_DB contains a collection of computationally predicted transcription factor binding sites in gamma-proteobacterial genomes [12]. RegTransBase is a manually curated database of regulatory interactions in prokaryotes, which contains data on the regulation of about 39041 genes in 531 organisms [13]. SwissRegulon is a database containing genome-wide annotations of regulatory sites produced using multiple alignments of orthologous intergenic regions from related genomes and known sites from the literature, and ChIP-on-chip binding data [14]. However, with the increase in the number of newly sequenced genomes, it is difficult to manually curate the functional elements for them, especially for the organisms that have not been studied in detail experimentally.

The available curated databases of transcriptional regulatory regions have been extensively used to train most of the well known promoter and DNA binding site prediction algorithms developed based on sequence motifs [15-24] as well as those using structure based properties of DNA [25-30]. There are also several other databases and servers which contain computationally derived information about distribution of transcription factors in bacterial genomes [31-33]. However, none of these databases cover the entire taxonomic diversity of prokaryotic genomes and the predictions have not been validated on a genomic scale, nor do they identify promoter regions for RNA genes. Hence, this remains an important lacuna for genomic and proteomic research in microbiology.

Here we describe PromBase, a web resource that has been constructed to provide the prediction and evaluation of promoter regions in a coherent manner, so that the user can browse and search each entry or download all the predicted promoter regions for any microbial genome. Apart from being a database for putative promoter regions, PromBase provides extensive information related to other genomic features such as base composition in various intergenic and coding regions, CDS-skew and CG/ TA skew along the genomic length, as well as DNA sequence dependent structural properties such as stability, curvature and bendability in the vicinity of promoter regions $(-500$ to +500 w.r.t TLSs). Stability profile can also be viewed for a $1001 \mathrm{nt}$ spanning region with respect to TLS of each individual gene displayed in the genome browser, along with the predicted promoter regions. Hence, this database can serve as an important resource for the molecular biology community to access genome related information and facilitate planning of experiments for reliable promoter regions.

\section{Database content}

The genome sequence for all microbial genomes was downloaded from NCBI ftp:/ftp.ncbi.nih.gov/genomes/ Bacteria/. Lower relative stability of DNA sequence has been used to predict promoter regions [34,35]. The method has been incorporated into an algorithm called 'PromPredict' and generalized to predict putative promoter regions in any given nucleotide sequence with a minimum length of $1000 \mathrm{nt}$. A detailed analysis of the 913 bacterial genomes, carried out after rationalizing the threshold values for identifying promoter regions in DNA sequences with varying $\mathrm{GC}$-content and assigning reliability scores to the predictions, has been recently reported [36]. A standalone version of PromPredict was used to develop 'PromBase', the database presented here, which displays the predicted promoter regions, along with their evaluation parameters, in a coherent manner, so that the users can browse and search each entry or download all the predicted promoter regions for any microbial genome. The average free energy profile for a $1001 \mathrm{nt}$ length sequence (spanning -500 to +500 w.r.t TLS) is also accessible for each gene displayed within the chosen variable size window. Download option is available for the predicted promoter data for all 913 microbial genomes.

In addition to acting as a resource for promoter annotation, PromBase also provides graphical representation of several other microbial genomic features such as

i. The GC-content distribution for all $1000 \mathrm{nt}$ long fragments (with $250 \mathrm{nt}$ overlap) in the genome along with their average free energy profiles.

ii. The cumulative CDS-skew $\left(C D S-\right.$ skew $\left.^{\mathrm{c}}\right)$ [37] as well as CG and TA skews $\left(\right.$ skew $\left.^{\mathrm{c}}\right)$ [38] for each genome.

iii. Percentage distribution, length and GC-content of different intergenic regions (tandem, divergent, convergent) as well as the protein and RNA coding regions, in each of the microbial genomes.

iv. Analysis of nucleotide composition and structural properties in promoter regions of protein coding genes

a. Distribution of $A, T, G, C$ nucleotides in the $101 \mathrm{nt}$ long sequences $(-80$ to +20 w.r.t TLS) and \% occurrence of tetranucleotides in the vicinity of TLS $(-150$ to +50 versus +200 to $+400 \mathrm{nt}$ region w.r.t TLS). 
b. \%GC and average free energy distribution for different regions $(-300$ to $-200,-80$ to +20 and +200 to +300$)$ in the vicinity of TLS.

c. CG and TA skews $[39,40]$ for $1001 \mathrm{nt}$ long sequences, spanning -500 to +500 w.r.t TLS.

d. Average free energy profile for $1001 \mathrm{nt}$ long sequences in the genome $(-500$ to +500 w.r.t TLS and all $1001 \mathrm{nt}$ long fragments with $250 \mathrm{nt}$ overlap).

e. Average curvature profile using dinucleotide parameters from crystal structure (CS Model [41]) and gel mobility (BHMT model [42]) data, for the $1001 \mathrm{nt}$ long sequences spanning TLSs.

f. Average bendability profile using DNase I sensitivity [43] and nucleosomal positioning preference [44] trinucleotide models, for the $1001 \mathrm{nt}$ long sequences spanning TLSs.

g. Z score plot for the DNA sequence dependent structural properties such as stability, bendability and curvature (only for CS model), for the 1001 nt long sequences spanning TLSs.

The analysis result for each feature listed above is shown in Figure 1 for E. coli K12 MG1655 strain. Details about the methods followed to calculate each of the above features have been provided in additional file 1 .

\section{Database construction}

The data is organized into PromBase using MySQL, a relational database management system that serves as the backend for storing data. The genome sequence data obtained from NCBI has been processed and stored into a table. The gene information for each genome and the detailed promoter prediction results for each genome are maintained in different tables. The calculated average free energy profile for the $1001 \mathrm{nt}$ length sequence in the vicinity of TLS of each gene $(-500$ to $+500 \mathrm{nt}$ region w.r.t TLS) is loaded into separate table per genome. These tasks were performed with variety of SQL queries embedded in PERL scripts. The relational database schema followed for PromBase query retrieval management is summarized in Additional File 2: Figure S1. Each table has a primary key entry and the reference to them is processed internally by CGI scripts for the web interface. The fields emphasized in italic bold face font in those tables (Additional File 2: Figure S1) were used as search keys in the web interface. The figures for genomic feature analysis of each genome have been generated using scripts written in MATLAB, which is a high-level technical computing language for algorithm development, data visualization, data analysis, and numeric computation. The genome browser view in the prediction result page as well as the average free energy profiles for each gene was generated using PERL
GD package. The web interface for PromBase is managed by a collection of HTML, cgi PERL scripts that do all the work, from querying the database upon user's request, to generating the dynamic web pages that form the interface. Apache is used as the web server.

\section{Utility}

\section{Database interface}

PromBase interface is well organized and managed at following levels. (a) Table of genome associated facts (NCBI reference table), which includes NCBI accession number, organism name with strain, size of the genome, GC composition, percentage of coding region and number of genes along with the gene product information, which were retrieved from NCBI for all 913 bacterial genomes. (b) Analysis of each genome for various features at different genomic regions as well as for sequence, and sequence dependent structural properties of DNA (as listed in previous section). (c) Detailed search results for predicted promoter regions, along with gene information, within a variable size window selected by the user, which is displayed in genome browser view. The database also correlates the predicted promoter regions with gene information in terms of true positive and false positive, depending on their location and the extensive analysis results are represented pictorially for each genome. (d) Tabulation of promoter prediction results along with the gene table that lies within the variable size window. (e) Average free energy profile for a region spanning -500 to +500 w.r.t TLS of each gene that is tabulated. Figure 2 illustrates the web interface maintained for PromBase.

\section{Query and data retrieval hierarchy}

At the first stage, an organism name is the initial query to PromBase. Upon the submission of organism name, PromBase web interface leads to a second stage query page. This page requests input for either gene name specific search or position specific search within a genomic region. The second stage query page also contains the genome feature analysis results for the query genome (Figure 1). The predicted promoter regions, along with gene information found within a variable size window selected by the user, are displayed in a genome browser view in the third stage interface (Figure 3). The exact position, nucleotide sequence and associated gene ID for the predicted promoters are also given in the tabular form in fourth stage interface (Additional File 2: Figure S2). A search using the GenBank gene ID is available at fourth stage interface, for viewing the average free energy (AFE) profile and predicted promoter region in the 500 nt flanking region, with respect to the translation start site (TLS) of the genes displayed in the genome browser 


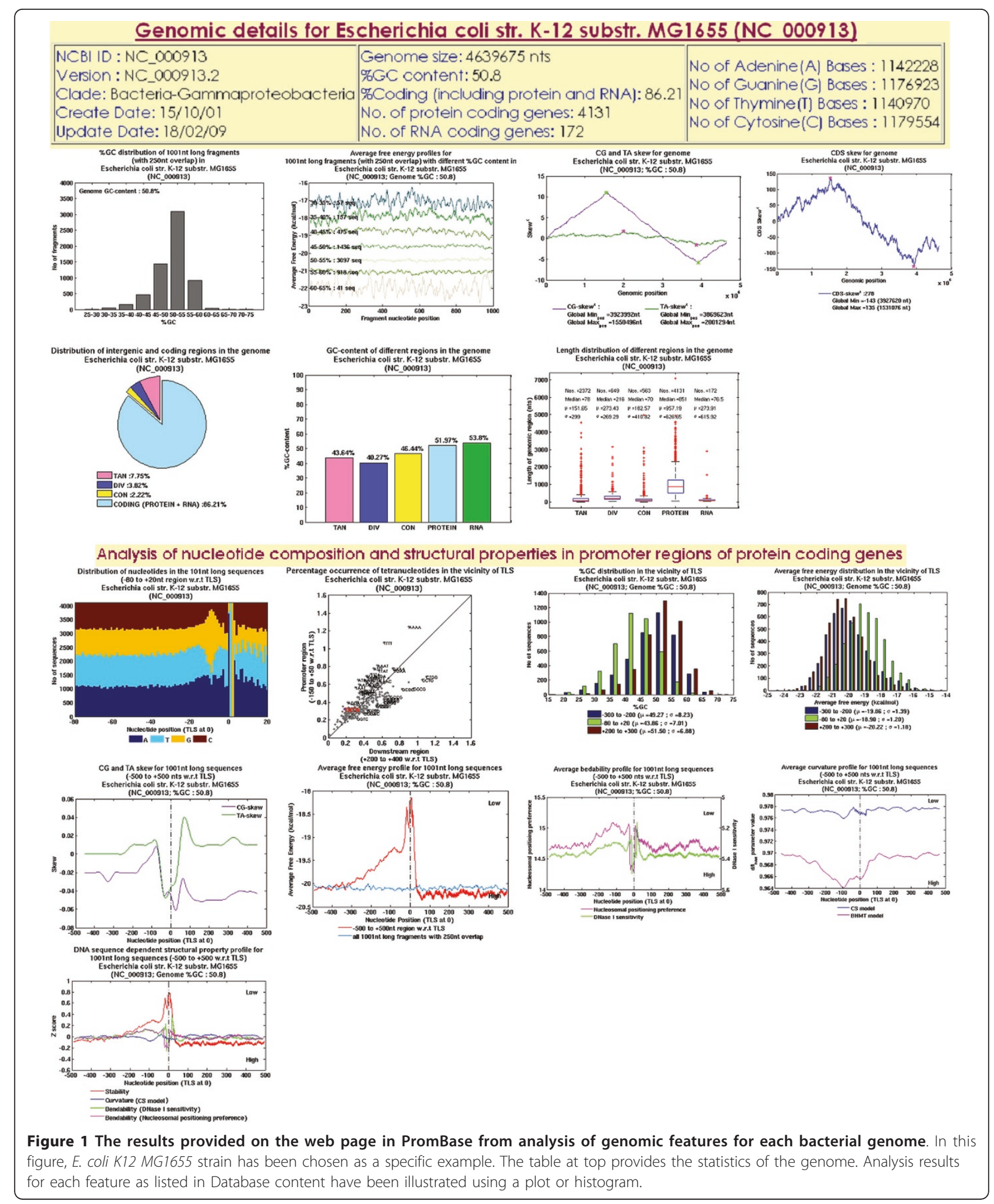




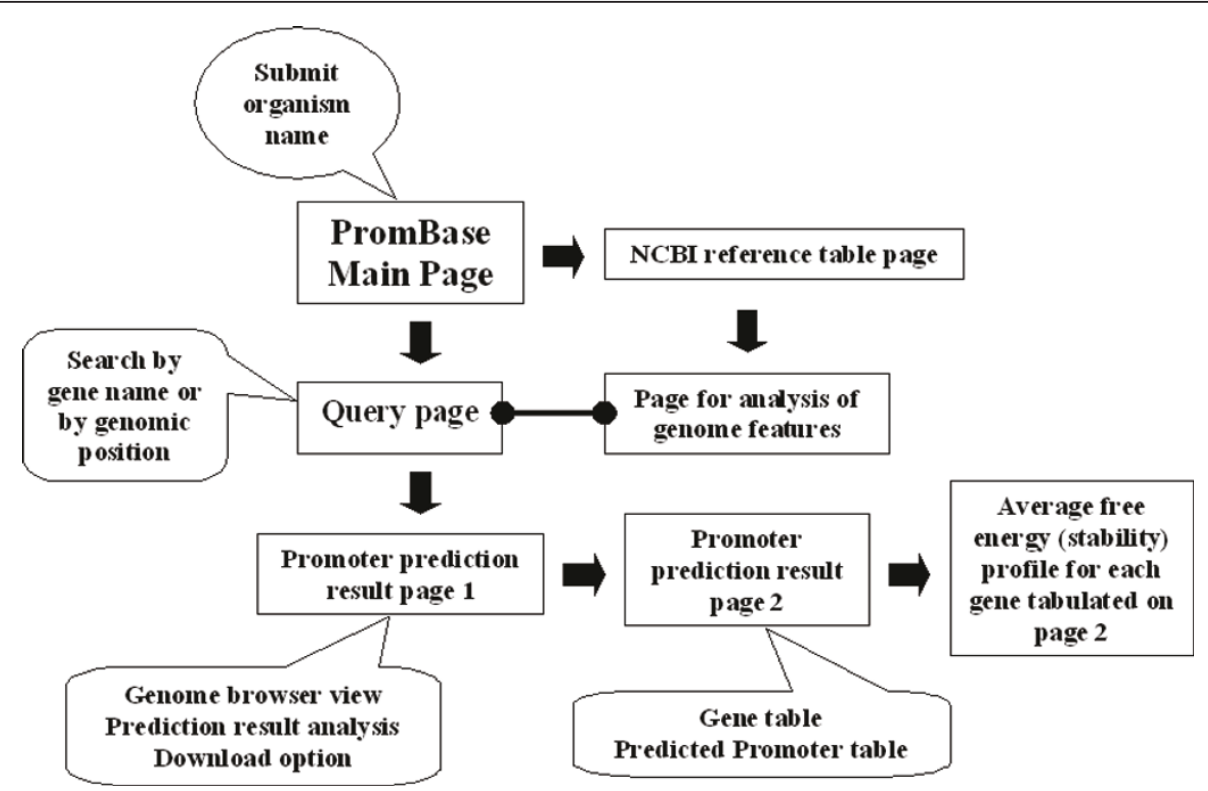

Figure 2 PromBase web interface. Rectangular box indicates a web page. Block arrows indicate the page transition caused by an action. Callouts give a simple explanation for information content availability at each page. Round headed line shows interlock between the information availability.

view (Additional File 2: Figure S2). In future, other DNA sequence dependent structural property (such as curvature and bendability) profiles in the vicinity of TLS of each gene will also be included in PromBase.

\section{Discussion}

\section{Promoter annotation in PromBase}

As compared to the other databases available for prediction of regulatory elements (generally TFBS) in genomic sequences (discussed in introduction section), PromBase provides a large scale annotation of promoter regions in diverse prokaryotic genomes. Also, the predicted promoter regions have been classified into five different reliability levels (low, medium, high, very high and highest) based on the difference in their relative average free energy [36] and highlighted in PromBase using different colors. Using this reliability level classification scheme, users can design experiments with more confidence for the predictions with higher reliability levels. Within a predicted promoter region, the position corresponding to maximum difference in relative stability $\left(\mathrm{DE}_{\max }\right)$ has been highlighted, which can act as a reference position (if a predicted region is very long) for designing primers. Whole genome annotation for promoter regions and their quality, as well as distribution among various genomic regions has been analyzed extensively in terms of \% recall and \%precision [36] and the results are represented graphically in PromBase for each organism. The number of RNA genes with identified promoter regions has also been highlighted.

\section{Genome feature analysis in PromBase}

In addition to being a database for promoter prediction, PromBase also analyses and shows various other genomic features (listed in the Database content section). The sequence dependent DNA structural properties (stability, bendability and curvature), CG-skew and TA-skew which have been reported to have characteristic features in the vicinity of transcription start sites (TSS) $[26,37,39,45]$ have also been represented as plots with reference to translation start sites (TLS) for all microbial genomes. Since the TSS data is available only for a few genomes and the distance between TSS and TLS is generally small in prokaryotes, the prominent features can also be seen in a plot with respect to TLS. Comparison of the DNA sequence dependent structural property profiles in three microbial genomes (E. coli, B. subtilis and M. tuberculosis) with varying \% GC-content (50.8, 43.4 and 65.6 respectively) showed differences in the shape of their stability profiles [35]. However, irrespective of the genome GC-content a low stability peak was observed upstream of TLS in all systems. Detailed analysis of DNA structural property profiles (stability, bendability and curvature) for the above mentioned three systems revealed that stability could delineate promoter regions better than other properties (can be inferred from the Z-score plots for E. coli, B. subtilis and M. tuberculosis shown in PromBase) [46]. Thus PromBase can help the research community to compare and analyze the features present in the vicinity of TLS of various microbial genomes, from 


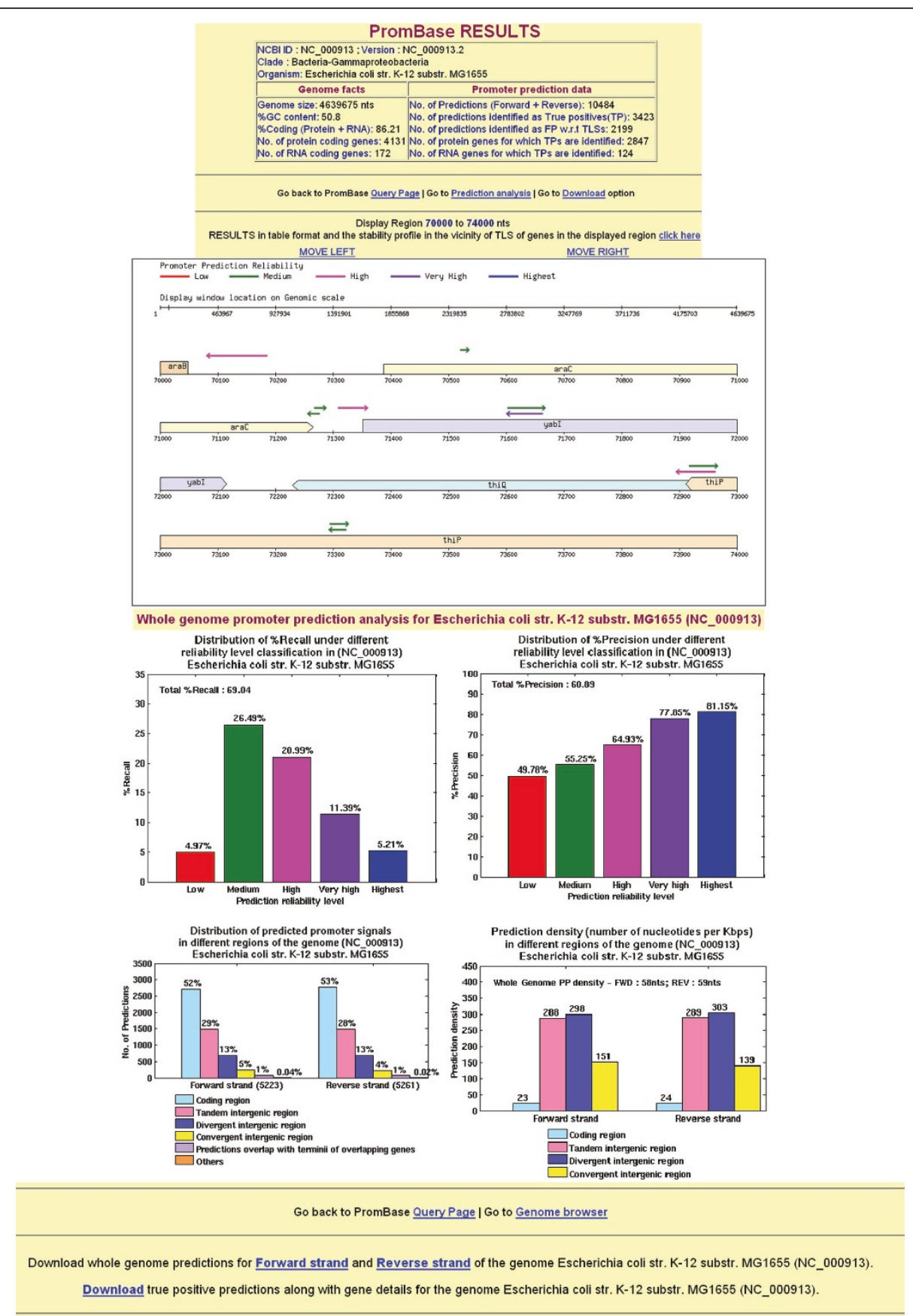

Figure 3 PromBase result page for prediction and analysis of promoter regions in E. coli $\mathbf{K 1 2}$ MG1655 strain. Table at top provides the statistics for the whole genome promoter prediction for E. coli. Genome browser view is shown for a position specific search centered on 72 $\mathrm{Kbp}$ of the genome with a flanking region of $2 \mathrm{Kbp}$. Histogram below illustrates the analysis of the prediction results in terms of \%recall and \% precision, as well as prediction distribution and density within various intergenic and coding regions.

different phyla or with different GC-content. Cumulative CG-skew and TA-skew $\left(\right.$ skew $\left.^{\mathrm{c}}\right)$ plotted for whole genome sequences can be used to determine the position of the origin of replication in bacterial species [47]. The shape of the CDS skew shown in PromBase for all microbial genomes could be helpful in observing the specific trends followed for the gene orientation throughout the genome, which has been suggested to be the main factor responsible for the observed nucleotide skews $[38,48]$. 


\section{Statistics of genome characteristics which are displayed in PromBase}

As there is rapid accumulation of bacterial genomes over a decade, prokaryotic genomes have been analyzed for their genomic features and their variation among phyla [1,49-51]. It is important to analyze the general genome characteristics (particularly the GC-content distribution) of all bacterial genomes for a better evaluation of prediction results from any method. Hence we have carried out a comprehensive and quantitative analysis of the genome features such as, genome size, \%GC-content, total number of genes, gene density and \%coding region for all 913 microbial genomes downloaded from NCBI. Figure 4 illustrates the statistics of the above mentioned features. A large number of genomes have $\sim 2 \mathrm{Mbp}$ and $\sim 5 \mathrm{Mbp}$ genome size (246 genomes within 1.5 to 2.5 bins and 161 genomes within 4.5 to 5.5 bins respectively in Figure 4A). Sorangium cellulosum 'So ce 56' (NC_010162) from Delta-proteobacteria phylum is the largest genome (13 $\mathrm{Mbp}$ ) with highest number of genes (9700). Candidatus Carsonella ruddii PV (NC_008512) which belongs to Gamma-proteobacteria phylum is the smallest genome (0.16 Mbp), has lowest \%GC content (16.6\%) and least number of genes (213). But it has highest gene density (1334 nucleotides per Mbps) and highest amount of \% coding region (97.3\%). The base composition of bacteria varies extensively between species. Anaeromyxobacter dehalogenans 2CP-C (NC_007760) genome (belonging to
Delta-proteobacteria) has highest amount of GC (74.9\%) which has been attributed to mutational bias $[52,53]$ while a maximum number of genomes (144 and 145) have their GC distribution within the range of $35-40 \%$ and $65-70 \%$ respectively (Figure $4 \mathrm{~B}$ ). The percentage of coding sequence in a genome is very much higher in prokaryotes as compared to eukaryotes. On an average $86.4 \%$ bacterial genome sequence is coding either for protein or for RNA. The \%coding region for the genomes Orientia tsutsugamushi str. Boryong (NC_009488; Phylum: Alphaproteobacteria), Mycobacterium leprae Br4923 and TN (NC_011896 and NC_002677; Phylum: Actinobacteria) is less as compared to the \%intergenic region in the respective genomes.

The number of overlapping genes is also high in prokaryotes due to the dense packing of genetic elements. Figure 4F shows the overlapping gene distribution in all microbial genomes. Since there is a tail towards the higher end, it leads to the mean value being higher (420 genes) and it is more meaningful to consider the median value (365 genes). It has been found that the increase in overlapping genes is caused more frequently by mutations at 3'-end of gene in closely related species and mutations at 5'-end of gene in distant species [54]. Another study had revealed that a large number of misannotations happened at 5'-end due to mispredictions of start codons among co-directional and divergently oriented genes [55] and a database has been developed to

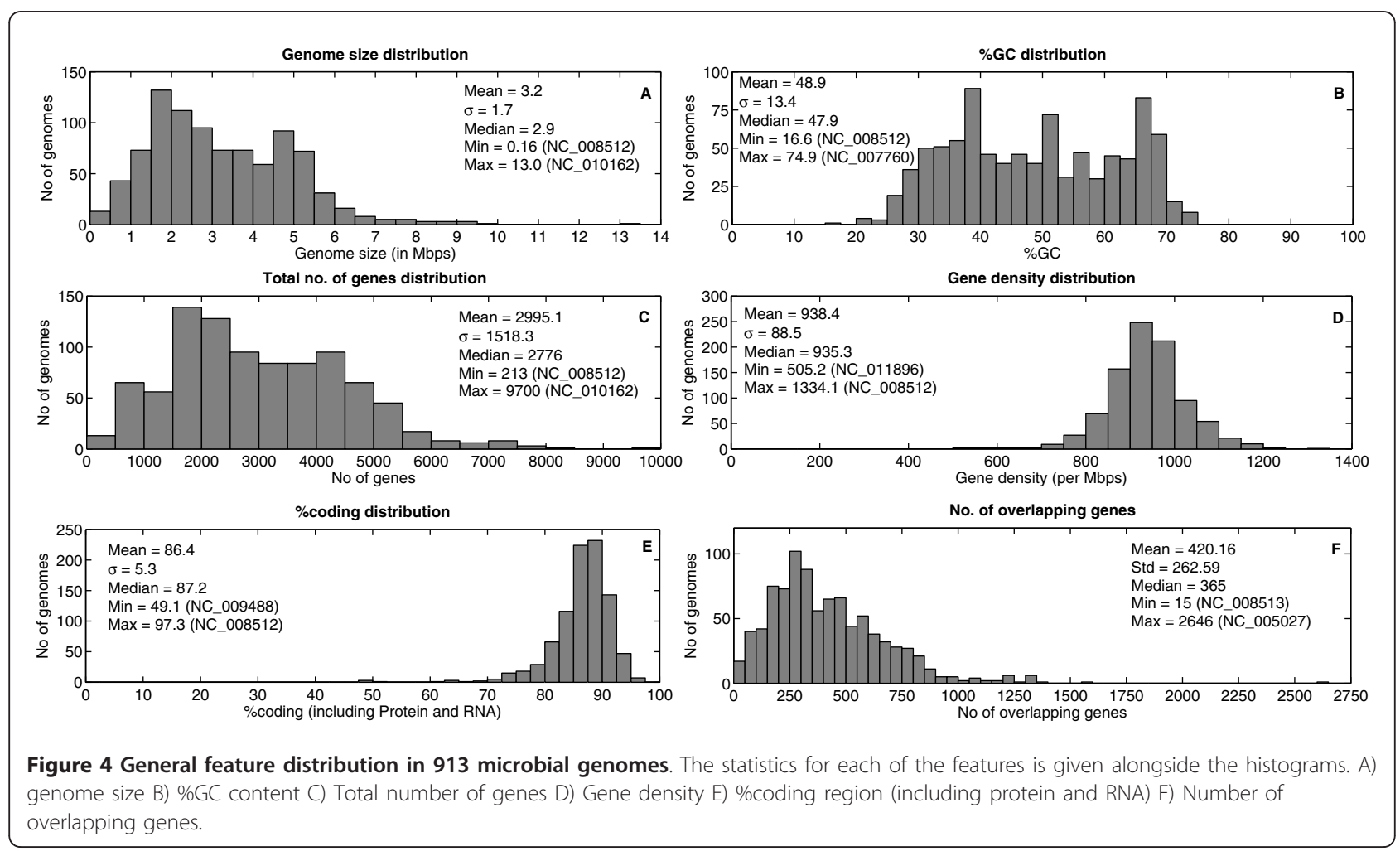


analyze the reliability of overlapping gene structures [56]. PromBase also provides a list of overlapping genes with a download option.

\section{Distribution of TAN, DIV, CON intergenic and CODING regions within microbial genomes and the conservation of their length and \%GC distribution}

The prokaryotic genomes consist largely of proteins genes and structural RNAs and only a small fraction constitutes the non-coding DNA. With the increase in genome sequence assembly of bacterial genomes, we have repeated the statistical analysis of the various types of intergenic regions in the bacterial genomes and the results are shown in PromBase for each genome. Figure 5 shows the conservation of relative lengths distribution and \%GC-content of TANDEM (TAN), DIVERGENT (DIV), CONVERGENT (CON) and CODING regions in all 913 bacterial genomes. In general, the trend of protein coding genes $>$ RNA genes $>$ DIV IR regions $>$ CON IR regions $>$ TAN IR regions (Figure $5 \mathrm{~A}$ ) is shown to be retained. The length of these intergenic spacers between genes are thought to be important since they are the sites for regulatory signals [57]. The average length of divergent intergenic region being longer as compared to the other intergenic regions might be essential as they contain upstream regulatory signals for two genes. The convergent gene length distribution has a tail for long CON IR, which is reflected in the standard deviation value (154.2), being almost equal to the mean (198.2). The DIV IR length distribution also has an extended tail towards the higher values, but the distribution is broader as compared to the CON IR length distribution, as showed earlier for 39 bacterial genomes [57]. Though the average length of TAN IR region is smaller it has higher spread than compared to other types of intergenic regions in microbial genomes (Figure 5B). This suggests that the number of genes that are transcribed together or the chances for the adjacent genes to form a gene cluster or an operon is high in bacterial genomes [58-61], in order to optimize the energy expenditure for the expression of highly expressed genes under specific growth conditions. Four microbial genomes have $\sim 50 \%$ genome comprising the intergenic region (Orientia tsutsugamushi str. Boryong, Mycobacterium leprae Br4923, Mycobacterium leprae TN, Sodalis glossinidius str. 'morsitans'). Comparative genome studies have revealed a drastic gene reduction and decay in Mycobacterium leprae genome by retaining only a minimal set of genes (less than half of the genome contain functional genes, while having abundant amount of pseudogenes) [62]. Analysis on accumulation of pseudogenes in Mycobacterium leprae genome has revealed the functional relevance of gene order within operons [63]. This study indicated that functionally less important genes have tendency to be located at the end of the operons, while more relevant genes tend to be located towards operon start. This particular genome has been shown as an extreme example for reductive evolution, the process by which large scale loss of gene function arises by inactivating the genes once their functions are no longer required in the highly specialized niches [64].

Figure $5 \mathrm{C}$ shows the \%GC-content distribution in all four regions. It is clearly seen that the regional rule, RNA gene $\% \mathrm{GC}>$ Protein gene $\% \mathrm{GC}>\mathrm{CON}$ IR $\% \mathrm{GC}>$ TAN IR \%GC > DIV IR \%GC which has been reported earlier for 183 genomes [65] is maintained, with 689 bacterial genomes satisfying the general regional rule. Among the remaining 224 genomes, 81 did not satisfy the first rule (i.e. \%GC coding > \%GC CON IR), 105 did not follow the second criteria (i.e. \%GC CON IR > \%GC TAN IR) and 22 did not follow the last rule (i.e. \%GC TAN IR > \%GC DIV IR). For 16 genomes two rules are not satisfied. The regional rule applies to a huge number of bacterial genomes and it has been suggested that it is correlated with DNA structural properties such as stability, bendability and curvature [65].

Thus PromBase gives an overview of a large number of nucleotide composition as well as structural properties for every microbial genome. There are a few other databases available for viewing genomic properties wherein the results are presented graphically as radial plots or 'atlas' and as a Z score plot for each structural property [66-68]. However, these databases do not analyze the characteristic features of different intergenic and coding regions in detail, as presented in PromBase, hence combining the information from these databases with PromBase will allow for a better understanding of genome information content.

\section{Conclusion}

PromBase can serve as a user friendly single point resource for the microbial genomic community to access information about several important genomic features for the genome of their interest. It helps to visualize the features present in the vicinity of TLS of various microbial genomes from different phyla or with different GC-content and also to explore the annotation of putative promoter regions, which could aid in transcriptional regulation of a gene. The web interface of PromBase is well organized and it also provides a download option for whole genome annotation of promoter regions. The free energy based classification scheme followed for categorizing the reliability of the predicted regions could help the experimentalists in designing their experiments. In addition to providing users a friendly input/output interface, PromBase gives a genome browser view for the annotated promoter regions in whole genome. 

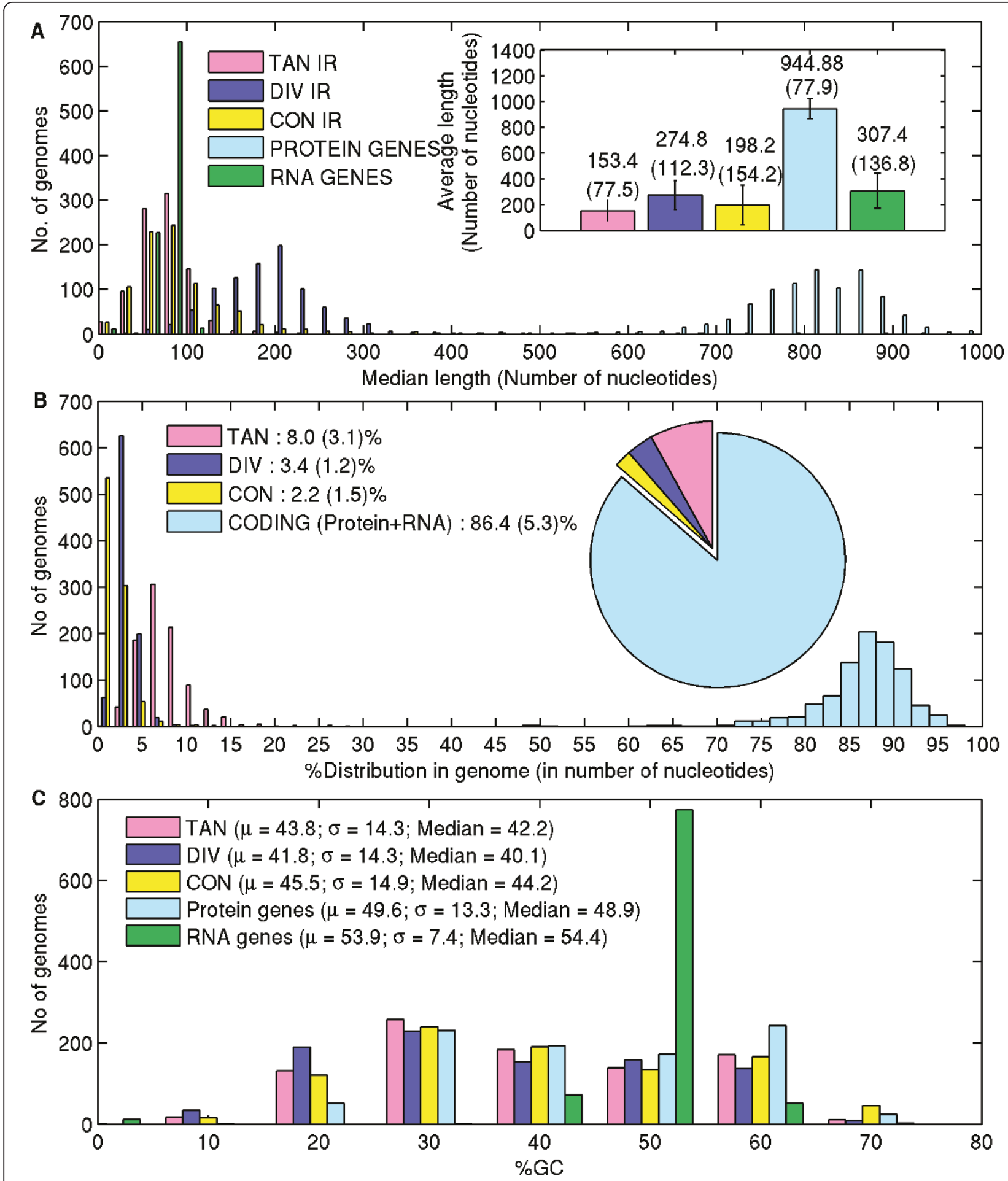

Figure 5 Analysis of TAN, DIV, CON intergenic regions and CODING regions. A) Length distribution of different regions in bacterial genomes. Inner bar chart shows the average length of different regions in all 913 bacterial genomes. The standard deviation for length of different regions is indicated as error bar (values are given in brackets). B) Percentage distribution of different intergenic and coding regions in bacterial genomes. Inner pie chart gives the overall average distribution in all bacterial genomes. The average value and the standard deviations (in bracket) are shown with the legend. RNA gene distribution is very small $(0.73 \%)$ as compared to others, hence it is not shown in the figure. C) GC-content distribution in different regions of bacterial genomes. The overall mean, standard deviation and median values are also given. 


\section{Availability and requirements}

Project Name: PromBase: A web resource for various genomic features and predicted promoters in prokaryotic genomes.

Project home page: http://nucleix.mbu.iisc.ernet.in/ prombase/

User side requirements: Any standard WWW browsers, such as firefox and internet explorer

Server side requirements:

Operating system: Linux.

Programming and scripting languages: HTML, MySQL, PERL, MATLAB

This database is freely accessible for all academic and non-academic users.

\section{Additional material}

Additional file 1: Method details. Contains the details of the methods followed for the calculation of each feature presented in PromBase.

Additional file 2: Figure S1 and Figure S2. Figure S1-Relational database schema used to construct PromBase; Figure S2 - PromBase results page for tabulation of promoter prediction results along with the gene table that lies within the variable size window of the genome browser view.

\section{List of abbreviations}

AFE: Average free energy; TSSs: Transcription start sites; TLSs: Translation start sites; CDS: Coding sequence.

\section{Acknowledgements}

This project was partially supported by grants received from Microsoft Corporation, USA and Department of Biotechnology, INDIA.

\section{Authors' contributions}

VR analyzed the data for general genome features displayed in PromBase, developed the database and wrote the manuscript. MB designed and supervised the study, corrected the manuscript and provided comments on the website construction and manuscript organization. All authors read and approved the final manuscript.

\section{Competing interests}

The authors declare that they have no competing interests.

Received: 27 January 2011 Accepted: 22 July 2011

Published: 22 July 2011

\section{References}

1. Lagesen K, Ussery DW, Wassenaar TM: Genome Update: The Thousandth Genome-A Cautionary Tale. Microbiology 2010, 156:603-608.

2. Hershberg R, Altuvia S, Margalit H: A survey of small RNA-encoding genes in Escherichia coli. Nucleic Acids Res 2003, 31:1813-1820.

3. Jacques PE, Gervais AL, Cantin M, Lucier JF, Dallaire G, Drouin G, Gaudreau L, Goulet J, Brzezinski R: MtbRegList, a database dedicated to the analysis of transcriptional regulation in Mycobacterium tuberculosis. Bioinformatics 2005, 21:2563-2565.

4. Keseler IM, Collado-Vides J, Gama-Castro S, Ingraham J, Paley S, Paulsen IT, Peralta-Gil M, Karp PD: EcoCyc: a comprehensive database resource for Escherichia coli. Nucleic Acids Res 2005, 33:D334-337
5. Makita Y, Nakao M, Ogasawara N, Nakai K: DBTBS: database of transcriptional regulation in Bacillus subtilis and its contribution to comparative genomics. Nucleic Acids Res 2004, 32:D75-77.

6. Salgado H, Gama-Castro S, Martinez-Antonio A, Diaz-Peredo E, SanchezSolano F, Peralta-Gil M, Garcia-Alonso D, Jimenez-Jacinto V, Santos-

Zavaleta A, Bonavides-Martinez C, Collado-Vides J: RegulonDB (version 4.0): transcriptional regulation, operon organization and growth conditions in Escherichia coli K-12. Nucleic Acids Res 2004, 32:D303-306.

7. Passalacqua KD, Varadarajan A, Ondov BD, Okou DT, Zwick ME, Bergman NH: Structure and complexity of a bacterial transcriptome. $J$ Bacteriol 2009, 191:3203-3211.

8. Wurtzel O, Sapra R, Chen F, Zhu Y, Simmons BA, Sorek R: A single-base resolution map of an archaeal transcriptome. Genome Res 2010, 20:133-141.

9. Filiatrault MJ, Stodghill PV, Bronstein PA, Moll S, Lindeberg M, Grills G, Schweitzer P, Wang W, Schroth GP, Luo S, et al: Transcriptome analysis of Pseudomonas syringae identifies new genes, noncoding RNAs, and antisense activity. J Bacteriol 2010, 192:2359-2372.

10. Sharma CM, Hoffmann S, Darfeuille F, Reignier J, Findeiss S, Sittka A, Chabas S, Reiche K, Hackermuller J, Reinhardt R, et al: The primary transcriptome of the major human pathogen Helicobacter pylori. Nature 2010, 464:250-255

11. Munch R, Hiller K, Barg H, Heldt D, Linz S, Wingender E, Jahn D: PRODORIC: prokaryotic database of gene regulation. Nucleic Acids Res 2003, 31:266-269.

12. Gonzalez AD, Espinosa V, Vasconcelos AT, Perez-Rueda E, Collado-Vides J: TRACTOR_DB: a database of regulatory networks in gammaproteobacterial genomes. Nucleic Acids Res 2005, 33:D98-102.

13. Kazakov AE, Cipriano MJ, Novichkov PS, Minovitsky S, Vinogradov DV, Arkin A, Mironov AA, Gelfand MS, Dubchak I: RegTransBase-a database of regulatory sequences and interactions in a wide range of prokaryotic genomes. Nucleic Acids Res 2007, 35:D407-412.

14. Pachkov M, Erb I, Molina N, van Nimwegen E: SwissRegulon: a database of genome-wide annotations of regulatory sites. Nucleic Acids Res 2007, 35: D127-131.

15. Carlson JM, Chakravarty A, DeZiel CE, Gross RH: SCOPE: a web server for practical de novo motif discovery. Nucleic Acids Res 2007, 35:W259-264.

16. Chakravarty A, Carlson JM, Khetani RS, Gross RH: A novel ensemble learning method for de novo computational identification of DNA binding sites. BMC Bioinformatics 2007, 8:249.

17. Gordon JJ, Towsey MW, Hogan JM, Mathews SA, Timms P: Improved prediction of bacterial transcription start sites. Bioinformatics 2006, 22:142-148.

18. Gordon L, Chervonenkis AY, Gammerman AJ, Shahmuradov IA, Solovyev W: Sequence alignment kernel for recognition of promoter regions. Bioinformatics 2003, 19:1964-1971.

19. Jacques PE, Rodrigue S, Gaudreau L, Goulet J, Brzezinski R: Detection of prokaryotic promoters from the genomic distribution of hexanucleotide pairs. BMC Bioinformatics 2006, 7:423.

20. Mann S, Li J, Chen YP: A pHMM-ANN based discriminative approach to promoter identification in prokaryote genomic contexts. Nucleic Acids Res 2007, 35:e12.

21. Reese MG: Application of a time-delay neural network to promoter annotation in the Drosophila melanogaster genome. Comput Chem 2001, 26:51-56.

22. Solovyev W, Shahmuradov IA: PromH: Promoters identification using orthologous genomic sequences. Nucleic Acids Res 2003, 31:3540-3545.

23. Studholme DJ, Dixon R: Domain architectures of sigma54-dependent transcriptional activators. J Bacteriol 2003, 185:1757-1767.

24. Dekhtyar M, Morin A, Sakanyan V: Triad pattern algorithm for predicting strong promoter candidates in bacterial genomes. BMC Bioinformatics 2008, 9:233.

25. Du Z, Zhao Y, Li N: Genome-wide analysis reveals regulatory role of G4 DNA in gene transcription. Genome Res 2008, 18:233-241.

26. Kanhere A, Bansal M: Structural properties of promoters: similarities and differences between prokaryotes and eukaryotes. Nucleic Acids Res 2005, 33:3165-3175.

27. Rawal P, Kummarasetti VB, Ravindran J, Kumar N, Halder K, Sharma R, Mukerji M, Das SK, Chowdhury S: Genome-wide prediction of G4 DNA as regulatory motifs: role in Escherichia coli global regulation. Genome Res 2006, 16:644-655. 
28. Wang H, Benham $\mathrm{CJ}$ : Promoter prediction and annotation of microbial genomes based on DNA sequence and structural responses to superhelical stress. BMC Bioinformatics 2006, 7:248

29. Yadav VK, Abraham JK, Mani P, Kulshrestha R, Chowdhury S: QuadBase: genome-wide database of G4 DNA-occurrence and conservation in human, chimpanzee, mouse and rat promoters and 146 microbes. Nucleic Acids Res 2008, 36:D381-385.

30. Gan Y, Guan J, Zhou S: A pattern-based nearest neighbor search approach for promoter prediction using DNA structural profiles. Bioinformatics 2009, 25:2006-2012.

31. Martinez-Bueno M, Molina-Henares AJ, Pareja E, Ramos JL, Tobes R: BacTregulators: a database of transcriptional regulators in bacteria and archaea. Bioinformatics 2004, 20:2787-2791.

32. Pareja E, Pareja-Tobes P, Manrique M, Pareja-Tobes E, Bonal J, Tobes R: ExtraTrain: a database of Extragenic regions and Transcriptional information in prokaryotic organisms. BMC Microbiol 2006, 6:29.

33. Yellaboina S, Seshadri J, Kumar MS, Ranjan A: PredictRegulon: a web server for the prediction of the regulatory protein binding sites and operons in prokaryote genomes. Nucleic Acids Res 2004, 32:W318-320.

34. Kanhere A, Bansal M: A novel method for prokaryotic promoter prediction based on DNA stability. BMC Bioinformatics 2005, 6:1.

35. Rangannan $\mathrm{V}$, Bansal M: Relative stability of DNA as a generic criterion for promoter prediction: whole genome annotation of microbial genomes with varying nucleotide base composition. Mol Biosyst 2009, 5:1758-1769.

36. Rangannan V, Bansal M: High Quality Annotation of Promoter Regions for 913 Bacterial Genomes. Bioinformatics 2010, 26:3043-3050.

37. Touchon M, Nicolay S, Arneodo A, d'Aubenton-Carafa Y, Thermes C Transcription-coupled TA and GC strand asymmetries in the human genome. FEBS Lett 2003, 555:579-582.

38. Nikolaou C, Almirantis Y: A study on the correlation of nucleotide skews and the positioning of the origin of replication: different modes of replication in bacterial species. Nucleic Acids Res 2005, 33:6816-6822.

39. Fujimori S, Washio T, Tomita M: GC-compositional strand bias around transcription start sites in plants and fungi. BMC Genomics 2005, 6:26.

40. Lobry JR: Asymmetric substitution patterns in the two DNA strands of bacteria. Mol Biol Evol 1996, 13:660-665

41. Bansal M: Structural variations observed in DNA crystal structures and their implications for protein-DNA interactions. Biological structure and Dynamics, Proceedings of the Ninth Conversation 1996, I:121-134.

42. Bolshoy A, McNamara P, Harrington RE, Trifonov EN: Curved DNA without A-A: experimental estimation of all 16 DNA wedge angles. Proc Natl Acad Sci USA 1991, 88:2312-2316.

43. Brukner I, Sanchez R, Suck D, Pongor S: Trinucleotide models for DNA bending propensity: comparison of models based on DNasel digestion and nucleosome packaging data. J Biomol Struct Dyn 1995, 13:309-317.

44. Satchwell SC, Drew HR, Travers AA: Sequence periodicities in chicken nucleosome core DNA. J Mol Biol 1986, 191:659-675.

45. Abeel T, Saeys Y, Bonnet E, Rouze P, Van de Peer Y: Generic eukaryotic core promoter prediction using structural features of DNA. Genome Res 2008, 18:310-323.

46. Rangannan V, Bansal M: Analysis of DNA sequence dependent structural properties in prokaryotic genomes. ECCB10; Ghent, Belgium 2010, (Poster No. E-33).

47. Tillier ER, Collins RA: The contributions of replication orientation, gene direction, and signal sequences to base-composition asymmetries in bacterial genomes. J Mol Evol 2000, 50:249-257.

48. Necsulea A, Lobry JR: A new method for assessing the effect of replication on DNA base composition asymmetry. Mol Biol Evol 2007, 24:2169-2179

49. Bentley SD, Parkhill J: Comparative genomic structure of prokaryotes. Annu Rev Genet 2004, 38:771-792.

50. Binnewies TT, Motro Y, Hallin PF, Lund O, Dunn D, La T, Hampson DJ, Bellgard M, Wassenaar TM, Ussery DW: Ten years of bacterial genome sequencing: comparative-genomics-based discoveries. Funct Integr Genomics 2006, 6:165-185.

51. Koonin EV, Wolf Yl: Genomics of bacteria and archaea: the emerging dynamic view of the prokaryotic world. Nucleic Acids Res 2008, 36:6688-6719.

52. Cox EC, Yanofsky C: Altered base ratios in the DNA of an Escherichia coli mutator strain. Proc Natl Acad Sci USA 1967, 58:1895-1902.
53. Sueoka N: On the genetic basis of variation and heterogeneity of DNA base composition. Proc Natl Acad Sci USA 1962, 48:582-592.

54. Fukuda $Y$, Nakayama $Y$, Tomita M: On dynamics of overlapping genes in bacterial genomes. Gene 2003, 323:181-187.

55. Palleja A, Harrington ED, Bork P: Large gene overlaps in prokaryotic genomes: result of functional constraints or mispredictions? BMC Genomics 2008, 9:335.

56. Palleja A, Reverter T, Garcia-Vallve S, Romeu A: PairWise Neighbours database: overlaps and spacers among prokaryote genomes. BMC Genomics 2009, 10:281.

57. Rogozin IB, Makarova KS, Natale DA, Spiridonov AN, Tatusov RL, Wolf YI, Yin J, Koonin EV: Congruent evolution of different classes of non-coding DNA in prokaryotic genomes. Nucleic Acids Res 2002, 30:4264-4271.

58. Salgado H, Moreno-Hagelsieb G, Smith TF, Collado-Vides J: Operons in Escherichia coli: genomic analyses and predictions. Proc Natl Acad Sci USA 2000, 97:6652-6657.

59. Zhou D, Yang R: Global analysis of gene transcription regulation in prokaryotes. Cell Mol Life Sci 2006, 63:2260-2290.

60. Ermolaeva MD, White O, Salzberg SL: Prediction of operons in microbial genomes. Nucleic Acids Res 2001, 29:1216-1221.

61. Price MN, Huang KH, Alm EJ, Arkin AP: A novel method for accurate operon predictions in all sequenced prokaryotes. Nucleic Acids Res 2005, 33:880-892.

62. Vissa VD, Brennan PJ: The genome of Mycobacterium leprae: a minimal mycobacterial gene set. Genome Biol 2001, 2, REVIEWS1023.

63. Muro EM, Mah N, Moreno-Hagelsieb G, Andrade-Navarro MA: The pseudogenes of Mycobacterium leprae reveal the functional relevance of gene order within operons. Nucleic Acids Res 2010, 39:1732-1738.

64. Eiglmeier K, Parkhill J, Honore N, Garnier T, Tekaia F, Telenti A, Klatser P, James KD, Thomson NR, Wheeler PR, et al: The decaying genome of Mycobacterium leprae. Lepr Rev 2001, 72:387-398.

65. Mitchison G: The regional rule for bacterial base composition. Trends Genet 2005, 21:440-443.

66. Hallin PF, Ussery DW: CBS Genome Atlas Database: a dynamic storage for bioinformatic results and sequence data. Bioinformatics 2004, 20:3682-3686.

67. Zhang CT, Zhang R, Ou HY: The Z curve database: a graphic representation of genome sequences. Bioinformatics 2003, 19:593-599.

68. Stothard P, Van Domselaar G, Shrivastava S, Guo A, O'Neill B, Cruz J, Ellison M, Wishart DS: BacMap: an interactive picture atlas of annotated bacterial genomes. Nucleic Acids Res 2005, 33:D317-320.

doi:10.1186/1756-0500-4-257

Cite this article as: Rangannan and Bansal: PromBase: a web resource for various genomic features and predicted promoters in prokaryotic genomes. BMC Research Notes 2011 4:257.

\section{Submit your next manuscript to BioMed Central and take full advantage of:}

- Convenient online submission

- Thorough peer review

- No space constraints or color figure charges

- Immediate publication on acceptance

- Inclusion in PubMed, CAS, Scopus and Google Scholar

- Research which is freely available for redistribution

Submit your manuscript at www.biomedcentral.com/submit
C Biomed Central 